\title{
Three-dimensional fluid simulations of a simple magnetized toroidal plasma
}

\author{
Paolo Ricci ${ }^{1}$, B.N. Rogers ${ }^{2 *}$ \\ ${ }^{1}$ Centre de Recherches en Physique des Plasmas - École Polytechnique Fédérale de Lausanne, \\ Association EURATOM-Confédération Suisse, CH-1015 Lausanne, Switzerland \\ ${ }^{2}$ Department of Physics and Astronomy, \\ Dartmouth College, Hanover NH 03755
}

(Dated: August 13, 2009)

\begin{abstract}
Three-dimensional fluid simulations are performed in a simple magnetized toroidal plasma, in which vertical and toroidal magnetic fields create helicoidal magnetic field lines that terminate on the torus vessel. The simulations are carried out in the three-dimensional flux tube that wraps around the torus and are able to describe both interchange and drift-wave dynamics. The presence of different turbulence regimes is pointed out; in particular, it is shown that turbulence can be quenched by either a sufficiently large plasma source, or a sufficiently small vertical field, or a sufficiently high parallel resistivity. The simulations considered herein reveal that interchange turbulence dominates over the drift-wave dynamics. Considering the experimental observations, we finally discuss the limitations of the present model.
\end{abstract}

*Electronic address: paolo.ricci@epfl.ch, barrett.rogers@dartmouth.edu 


\section{INTRODUCTION}

We describe plasma dynamics in a simple magnetized torus (SMT), a configuration characterized by a toroidal magnetic field, $B_{\phi}$, superposed on a vertical magnetic field, $B_{v}$, resulting in helicoidal field lines terminating on the torus vessel $[1,2]$. As in the scrape-off layer of magnetic fusion devices, the SMT features open field lines, magnetic curvature, and plasma gradients, however in a environment that allows detailed experimental diagnostics, wide parameter scans, and a simplified setup to develop a physical understanding of the plasma dynamics and to perform a detailed comparison between simulations and experiments $[3,4]$.

Two main instabilities are potentially active in the SMT: the interchange instability, characterized by $k_{\|}=0$ and driven by the curvature and pressure gradient, and a $k_{\|} \neq 0$ instability, destabilized in the presence of a density gradient by resistivity, electron mass, and Landau dampingand affected by magnetic curvature [5]. Thus far, our theoretical investigations carried out in the SMT configuration have mainly focused on the interchangedriven turbulent regime. To describe turbulence in this regime, due to the $k_{\|}=0$ character of the interchange instability, a relatively simple two-dimensional model can be considered in a domain perpendicular to the field lines. The two-dimensional simulations in Ref. [6] show the presence of two turbulent regimes, roughly analogous to the low and high confinement modes in tokamaks, and denoted as L- and H-mode. The L-mode is characterized by weak shear flow effects and interchange-driven density and heat transport from the high field side of the SMT, where plasma is produced, to the low field side of the machine. The H-mode, accessed for sufficiently high temperature source strengths and low $B_{v}$, is characterized by a strong shear flow that limits perpendicular transport, steepening and peaking the plasma pressure profiles.

In the present paper we perform three-dimensional fluid simulations of a SMT that extend the two-dimensional simulations in Ref. [6] by taking into account the $k_{\|} \neq 0$ physics. The plasma dynamics are simulated in a flux tube that wraps around the SMT. The main two

findings of the present paper are: first, different turbulent regimes can be achieved by varying the plasma parallel resistivity, $\nu$, plasma source strength, $S_{0}$, and $B_{v}$ strength. The existence of the previously found L- and H-mode is confirmed, and a new turbulent regime, dubbed $\nu \mathrm{H}$-mode, is observed for sufficiently high values of $\nu$. As in the case of the H-mode, 
the $\nu \mathrm{H}$-mode is dominated by a strong shear flow that limits the perpendicular transport. However, its structure is inherently three-dimensional and its existence cannot be revealed by two-dimensional simulations. Second, the simulations explored show that drift waves do not play a major role in the plasma dynamics. Indeed, while the linear theory shows that the importance of $k_{\|} \neq 0$ instabilities is enhanced at steep plasma profiles, nonlinearly we observe that a strong shear flow arises at steep profiles that nonlinearly quench plasma turbulence.

The two-dimensional simulations considered a domain covering the full radial extension of the SMT and, because of the flute character of the interchange mode, a vertical extension equal to $\Delta=2 \pi R B_{v} / B_{\phi}=L_{v} / N$, i.e. the return distance of a field line in the poloidal plane ( $R$ and $L_{v}$ denote the major radius and the height of the SMT, $N$ the number of field line turns in the device). Periodic boundary conditions were thus applied in the vertical direction, which effectively neglect vessel effects on the perpendicular dynamics. The threedimensional simulations presented here consider a flux tube whose dimensions are the full SMT radial extension, $L_{c}=2 \pi N R$ in the parallel direction, and $\Delta$ in the vertical direction. Following the two-dimensional simulations, we maintain periodic boundary conditions in the vertical direction. The physical validity of such boundary conditions, which in our earlier two-dimensional work were motivated by the assumption that $k_{\|}=0$ interchangelike fluctuations dominate the system, as well as the neglect of vessel wall effects, are less clear in the three-dimensional case, in which $k_{\|} \neq 0$ modes can arise. We regard the present work as an incremental step toward global three-dimensional simulations, which will not only encompass $k_{\|} \neq 0$ modes but also include more realistic boundary conditions at the vessel walls. Further discussion of this point is given later.

The present paper is organized as follows. After the Introduction, in Sec. II the threedimensional model used for the simulation is presented. The linear modes are discussed in Sec. III. Section IV is focused on the nonlinear regimes: the L-mode, the H-mode, and the newly found $\nu \mathrm{H}$-mode are described. In Sec. $\mathrm{V}$, the transition threshold to a quenched turbulence regime is analytically evaluated and compared with simulation results. A final discussion follows in Sec. VI. In App. A we briefly summarize the main findings from the two-dimensional simulations in Ref. [6] related to purely interchange-driven dynamics. 


\section{MODEL}

Following the plasma parameters of the TORPEX experiment [1], we focus on the $T_{i} \ll T_{e}$ and $\beta \ll 1$ regime, and we use the drift-reduced Braginskii equations (e.g., see Ref. [7]) to describe the dynamics of density, $n$, electron temperature, $T_{e}$, potential, $\phi$, parallel electron and ion velocity, $V_{\| e}$ and $V_{\| i}$ :

$$
\begin{aligned}
& \frac{\partial n}{\partial t}=R[\phi, n]+2\left(n \frac{\partial T_{e}}{\partial y}+T_{e} \frac{\partial n}{\partial y}-n \frac{\partial \phi}{\partial y}\right) \\
& +D_{n} \nabla_{\perp}^{2} n-n \frac{\partial V_{\| e}}{\partial z}-V_{\| e} \frac{\partial n}{\partial z}+S_{n} \\
& \frac{\partial \nabla_{\perp}^{2} \phi}{\partial t}=R\left[\phi, \nabla_{\perp}^{2} \phi\right]-V_{\| i} \frac{\partial \nabla^{2} \phi}{\partial z}+2\left(\frac{T_{e}}{n} \frac{\partial n}{\partial y}+\frac{\partial T_{e}}{\partial y}\right) \\
& +\frac{1}{n} \frac{\partial j_{\|}}{\partial z}-\frac{\eta_{0 i}}{n}\left(2 \frac{\partial^{2} V_{\| i}}{\partial y \partial z}+\frac{\partial^{2} \phi}{\partial y^{2}}\right)+D_{\phi} \nabla_{\perp}^{4} \phi \\
& \frac{\partial T_{e}}{\partial t}=R\left[\phi, T_{e}\right]-V_{\| e} \frac{\partial T_{e}}{\partial z}+\frac{4}{3}\left(\frac{7}{2} T_{e} \frac{\partial T_{e}}{\partial y}+\frac{T_{e}^{2}}{n} \frac{\partial n}{\partial y}-T_{e} \frac{\partial \phi}{\partial y}\right) \\
& +D_{T} \nabla_{\perp}^{2} T_{e}+\frac{2}{3} \frac{T_{e}}{n} 0.71 \frac{\partial j_{\|}}{\partial z}-\frac{2}{3} T_{e} \frac{\partial V_{\| e}}{\partial z}+S_{T} \\
& \frac{m_{e}}{m_{i}} n \frac{\partial V_{\| e}}{\partial t}=\frac{m_{e}}{m_{i}} n R\left[\phi, V_{\| e}\right]-\frac{m_{e}}{m_{i}} n V_{\| e} \frac{\partial V_{\| e}}{\partial z} \\
& -T_{e} \frac{\partial n}{\partial z}+n \frac{\partial \phi}{\partial z}-1.71 n \frac{\partial T_{e}}{\partial z}+n \nu j_{\|}+\frac{4}{3} \eta_{0 e} \frac{\partial^{2} V_{\| e}}{\partial z^{2}} \\
& +\frac{2}{3} \eta_{0 e} \frac{\partial^{2} \phi}{\partial y \partial z}-\frac{2}{3} \frac{\eta_{0 e}}{n} \frac{\partial^{2} p_{e}}{\partial z \partial y}+D_{V_{e}} \nabla_{\perp}^{2} V_{\| e} \\
& n \frac{\partial V_{\| i}}{\partial t}=n R\left[\phi, V_{\| i}\right]-n V_{\| i} \frac{\partial V_{\| i}}{\partial z}-T_{e} \frac{\partial n}{\partial z}-n \frac{\partial T_{e}}{\partial z} \\
& +\frac{4}{3} \eta_{0, i} \frac{\partial^{2} V_{\| i}}{\partial z^{2}}+\frac{2}{3} \eta_{0, i} \frac{\partial^{2} \phi}{\partial y \partial z}+D_{V_{i}} \nabla_{\perp}^{2} V_{\| i}
\end{aligned}
$$

where $[a, b]=\partial_{x} a \partial_{y} b-\partial_{y} a \partial_{x} b, j_{\|}=n\left(V_{\| i}-V_{\| e}\right), D_{n}, D_{T}, D_{\phi}, D_{V e}$, and $D_{V i}$ are diffusion coefficients in the perpendicular plane, $\eta_{0 i}$ and $\eta_{0 e}$ are the ion and electron Braginskii viscosities, $\nu$ is the parallel resistivity, $S_{n}$ and $S_{T}$ are the density and temperature sources, and 
$m_{e} / m_{i}$ is the electron to ion mass ratio. The $x$ coordinate denotes the radial direction, $z$ is the coordinate parallel to the magnetic field, and $y$ is the direction perpendicular to $x$ and z. In Eqs. (2), we use the Boussinesq approximation for the polarization current [8]; also, we neglect thermal parallel conductivity in Eq. (3). We normalize $n$ to a reference density $n_{0}, T_{e}$ to $T_{e 0}, \phi$ to $T_{e 0} / e$, time $t$ to $R / c_{s 0}\left(c_{s 0}=\sqrt{T_{e 0} / m_{i}}\right)$, scale lengths to $\rho_{s 0}=c_{s 0} / \Omega_{c i}$ in the perpendicular directions and $R$ in the parallel direction.

The analysis presented here is carried out in a rectangular flux tube that follows the magnetic field lines and wraps around the SMT. The $x$ coordinate covers the full radial extension of the SMT; $y$ has extension $\Delta ; z$ extends over the connection length $L_{c}$, with metal plates perpendicular to the magnetic field lines at $z= \pm L_{c} / 2$. At the metal plates, we impose Bohm's sheath conditions by enforcing $V_{\| i}= \pm \sqrt{T_{e}}$ and $V_{\| e}= \pm \sqrt{T_{e}} \exp \left(\Lambda-\phi / T_{e}\right)$; on the other hand, for $n, \nabla^{2} \phi$, and $T_{e}$, no boundary conditions are necessary at $z= \pm L_{c} / 2$ since their respective evolution equations, Eqs. (1-3), involve only outflow convection along the parallel direction at the sheath. We use Dirichlet boundary conditions in the $x$ direction for all the fields and we apply periodic boundary conditions along the $y$ direction. In order to characterize the SMT magnetic configuration, we introduce the quantity $\sigma=R / L_{c}=$ $\Delta /\left(2 \pi L_{v}\right)$.

For the density and temperature sources, we use a profile that mimics the electron cyclotron (EC) and upper hybrid (UH) resonance layers in TORPEX, i.e. $S_{n}=S_{T}=$ $S_{0}\left\{S_{E C} \exp \left[-\left(x-x_{E C}\right)^{2} / \lambda_{E C}^{2}\right]+S_{U H} \exp \left[-\left(x-x_{U H}\right)^{2} / \lambda_{U H}^{2}\right]\right\}[9]$. This configurations represents the TORPEX scenario used for blob studies (see, e.g. Ref. [10]) characterized by an almost slab-like plasma source localized on the left part of the cross section. The source model adopted neglects the experimentally observed $z$ dependence of the source profile as well as the nonlinear dependence of the UH layer position on $n$ [9]. Numerical tests show that the simulation results discussed herein are insensitive to the details of the plasma source.

We remark that by integrating Eqs. (1-5) along the $z$ direction, the two-dimensional model used in Refs. $[3,6,11]$ can be deduced. The main results of the two-dimensional simulations are summarized in App. A.

Equations (1-5) are solved using a finite difference scheme. The $\mathbf{E} \times \mathbf{B}$ advective term is treated using the Arakawa advection scheme [12], while the parallel convective terms are discretized with a second order centered difference method. Time is advanced using a 
standard explicit Runge-Kutta stepping. Convergence tests have been performed with time and spatial discretization, and global conservation properties have also been checked.

In the simulation discussed in the present paper, we use $S_{U H}=1.5, S_{E C}=1, \lambda_{U H}=5$, $\lambda_{E C}=2.5, x_{U H}=15, x_{E C}=35, \Lambda=3, m_{i} / m_{e}=400, R=200$, and $L_{v}=64$. As shown in Table I, the dependence on the dissipative parameters is weak, and for the present simulations we use $D_{n}=D_{T}=D_{\phi}=D_{V e}=D_{V i}=1, \eta_{0 i}=\eta_{0 e}=0.1$. We focus on the dependence of the plasma dynamics on $\sigma, \nu$, and $S_{0}$.

\section{LINEAR MODES}

We linearize Eqs. (1-5), considering for simplicity a background density $\bar{n}=$ $n_{00}\left(1+x / L_{n}\right)$, electron temperature $\bar{T}_{e}(x)=T_{e 00}\left(1+x / L_{T}\right)$, potential $\bar{\phi}=\phi_{00}=\Lambda T_{e 00}$,

and parallel velocities $\bar{V}_{\| e}=\bar{V}_{\| i}=\bar{V}_{\|}(z)$ with $\bar{V}_{\|}\left( \pm L_{c} / 2\right)= \pm \sqrt{T_{e 00}}$. (The background plasma profile does not satisfy parallel force balance; however, the time scale of its evolution is slower than the typical linear growth rate of the instability by one order of magnitude or more.) The perturbed density is assumed to be in the form $\tilde{n}=\tilde{n}(z) \exp \left(\gamma t+i k_{y} y\right)$, and similar expressions are used for the other physical quantities. Neglecting dissipative terms, the linearized system can be written as:

$$
\begin{gathered}
\gamma \tilde{n}=i \omega_{*} \frac{n_{00}}{T_{e 00}} \tilde{\phi}+2 i \omega_{d}\left[\tilde{n}+\frac{n_{00}}{T_{e 00}}\left(\tilde{T}_{e}-\tilde{\phi}\right)\right]-\tilde{n} \frac{d \bar{V}_{\|}}{d z}-n_{00} \frac{d \tilde{V}_{\|}}{d z}-\bar{V}_{\|} \frac{d \tilde{n}}{d z} \\
-k_{y}^{2} \gamma \tilde{\phi}=2 i \omega_{d}\left(\frac{\tilde{n}}{n_{00}}+\frac{\tilde{T}_{e}}{T_{e 00}}\right)+k_{y}^{2} \bar{V}_{\|} \frac{d \tilde{\phi}}{d z}+\frac{1}{n_{00}} \frac{d \tilde{j}_{\|}}{d z} \\
\gamma \tilde{T}_{e}=i \eta \omega_{*} \tilde{\phi}+\frac{4}{3} i \omega_{d}\left(\frac{7}{2} \tilde{T}_{e}+\frac{T_{e 00}}{n_{00}} \tilde{n}-\tilde{\phi}\right)+\frac{2}{3}\left(0.71 \frac{T_{e 00}}{n_{00}} \frac{d \tilde{j}_{\|}}{d z}-T_{e 00} \frac{d \tilde{V}_{\|}}{d z}-\tilde{T}_{e} \frac{d \bar{V}_{\|}}{d z}\right)-\bar{V}_{\|} \frac{d \tilde{T}_{e}}{d z}(8) \\
\gamma \tilde{V}_{\| e}=-\bar{V}_{\|} \frac{d \tilde{V}_{\| e}}{d z}-\tilde{V}_{\| e} \frac{d \bar{V}_{\|}}{d z}+\frac{m_{i}}{m_{e}}\left(\nu \tilde{j}_{\|}+\frac{d \tilde{\phi}}{d z}-\frac{T_{e 00}}{n_{00}} \frac{d \tilde{n}}{d z}-1.71 \frac{d \tilde{T}_{e}}{d z}\right) \\
\gamma \tilde{V}_{\| i}=-\bar{V}_{\|} \frac{d \tilde{V}_{\| i}}{d z}-\tilde{V}_{\| i} \frac{d \bar{V}_{\|}}{d z}-\frac{T_{e 00}}{n_{00}} \frac{d \tilde{n}}{d z}-\frac{d \tilde{T}_{e}}{d z}
\end{gathered}
$$

being $\tilde{j}_{\|}=n_{00}\left(\tilde{V}_{\| i}-\tilde{V}_{\| e}\right), \eta=L_{n} / L_{T}$, and the curvature and diamagnetic frequencies defined as $\omega_{d}=k_{y} T_{00}$ and $\omega_{*}=k_{y} T_{00} R / L_{n}$, respectively. At $z= \pm L_{c} / 2$, linearizing the 
boundary conditions for the parallel velocity, one obtains $\tilde{V}_{\| i}= \pm \tilde{T}_{e} /\left(2 \sqrt{T_{e 00}}\right)$ and $\tilde{V}_{\| e}=$ $\pm\left[\tilde{T}_{e} / \sqrt{T_{e 00}}(\Lambda+1 / 2)-\tilde{\phi} / \sqrt{T_{e 00}}\right]$.

We first consider a homogeneous system in which analytic progress is possible, i.e. a infinitely long system in the parallel direction with $\bar{V}_{\| i}=\bar{V}_{\| e}=0$, such that sheath effects can be neglected. We can thus assume $\tilde{n}=\tilde{n} \exp \left(\gamma t+i k_{y} y+i k_{\|} z\right)$. By introducing the frequency $\omega_{\|}=k_{\|} \sqrt{T_{e 00}}$, the dispersion relation can be written as

$$
a_{0}+a_{1} \gamma+a_{2} \gamma^{2}+a_{3} \gamma^{3}+a_{4} \gamma^{4}+a_{5} \gamma^{5}=0
$$

where

$$
\begin{aligned}
& a_{0}=i \omega_{\| \mid}^{4}\left[0.71 \omega_{*}\left(\frac{2}{3} \eta-1\right)-3.33 \omega_{d}\right] \\
& a_{1}=\frac{2}{3}\left[6.01 \omega_{\|}^{2}-10 i \omega_{d} \nu\right] \omega_{d}\left(2 \omega_{d}-\omega_{*}\right)+\left(\frac{5}{3}+0.34 k_{y}^{2}\right) \omega_{\|}^{4}- \\
& \left(6.67 \frac{m_{e}}{m_{i}} \omega_{d}+\frac{10}{3} i k_{y}^{2} \nu-3.33 \frac{m_{e}}{m_{i}} \omega_{*}\right) \omega_{d} \omega_{\|}^{2} \\
& a_{2}=-\frac{1}{3} i\left\{40 \frac{m_{e}}{m_{i}} \omega_{d}^{3}-20\left[i\left(k_{y}^{2}-1\right) \nu+\frac{m_{e}}{m_{i}} \omega_{*}\right] \omega_{d}^{2}\right. \\
& \left.+2\left[\left(6.01 k_{y}^{2}-2.84\right) \omega_{\|}^{2}-3 i(\eta+1) \nu \omega_{*}\right] \omega_{d}+3(1.71 \eta+1) \omega_{\|}^{2} \omega_{*}\right\}+ \\
& \frac{5}{3} \omega_{\|}^{2} \nu k_{y}^{2}-\frac{10}{3} i \omega_{d} \omega_{\|}^{2} \frac{m_{e}}{m_{i}}+i(1+\eta) \omega_{\|}^{2} \frac{m_{e}}{m_{i}} \omega_{*} \\
& a_{3}=\frac{1}{3}\left\{20\left(1-k_{y}^{2}\right) \frac{m_{e}}{m_{i}} \omega_{d}^{2}-\left[20 i \nu k_{y}^{2}+6 \frac{m_{e}}{m_{i}}(\eta+1) \omega_{*}\right] \omega_{d}+\left[\left(8.85+1.42 \frac{m_{e}}{m_{i}}\right) k_{y}^{2}+3\right] \omega_{\|}^{2}\right\} \\
& a_{4}=\frac{1}{3} k_{y}^{2}\left(3 \nu-20 i \omega_{d} \frac{m_{e}}{m_{i}}\right) \\
& a_{5}=k_{y}^{2} \frac{m_{e}}{m_{i}}
\end{aligned}
$$

Both the interchange and the drift-wave instabilities are contained by the dispersion relation (11). We start from the analysis of the interchange instability. In the case of flute modes, $\omega_{\|}=0$, Eq. (11) becomes $\left(m_{e} \gamma / m_{i}+\nu\right)\left(b_{0}+b_{1} \gamma+b_{2} \gamma^{2}+b_{3} \gamma^{3}\right)=0$, with $b_{0}=$ $20 i \omega_{d}^{2}\left(2 \omega_{d}-\omega_{*}\right) / 3, b_{1}=20\left(k_{y}^{2}-1\right) \omega_{d}^{2} / 3+2(\eta+1) \omega_{*} \omega_{d}, b_{2}=20 i \omega_{d} k_{y}^{2} / 3$, and $b_{3}=-k_{y}^{2}$. This dispersion relation corresponds to the one in Ref. [13] in the limit $T_{i}=0$. In particular, for $k_{y} \ll 1$, if one scales $\gamma \sim c_{s} / R$, the dispersion relation of the interchange mode is obtained, $k_{y}^{2} \gamma^{2}+20 \omega_{d}^{2} / 3-2(\eta+1) \omega_{*} \omega_{d}=0$. The interchange mode is destabilized by curvature and 

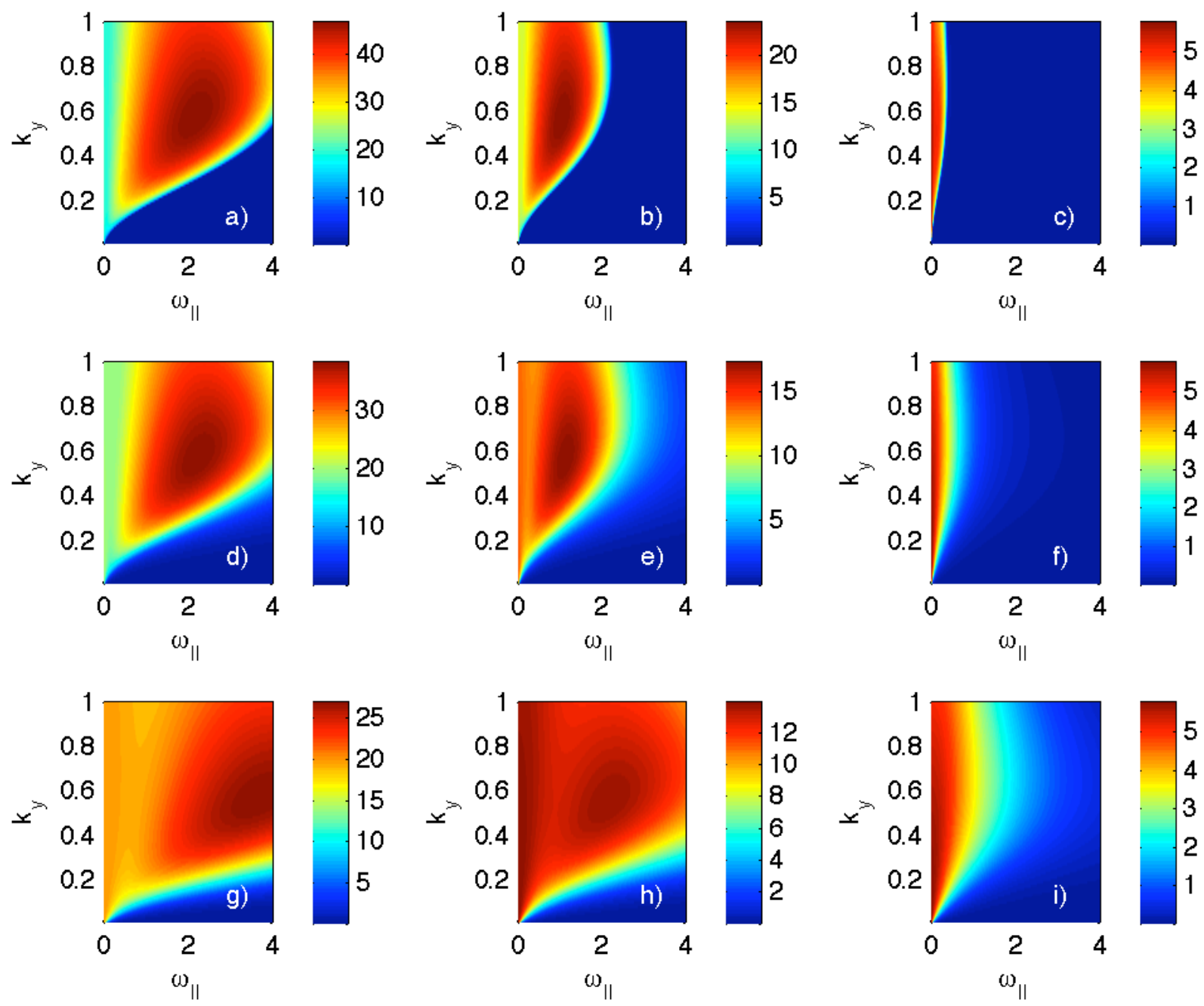

FIG. 1: (Color online) Linear growth rate of the most unstable mode evaluated by the dispersion relation in Eq. (11). $L_{n} / R=0.01$ (left panels: a, d, and g), $L_{n} / R=0.02$ (middle panels: b, e, and $\mathrm{g}$ ), and $L_{n} / R=0.1$ (right panels: c, f, and i); $\nu=0.001$ (upper panels: a-c), $\nu=0.1$ (middle panels: d-f), and $\nu=1$ (lower panels: g-i). It is $\eta=1, m_{i} / m_{e}=400$, and $\Lambda=3$.

pressure gradients, tends to grow on the longest allowed $y$ scale, has $k_{\|}=0$, and has a linear growth rate $\gamma \sim c_{s} / \sqrt{R L_{p}}$. We note that if one scales $\omega \sim \omega_{*} \sim \omega_{d}$, the dispersion relation for the entropy mode [13] is also obtained, $\gamma=10 i \omega_{d}\left(2 \omega_{d}-\omega_{*}\right) /\left[3(\eta+1) \omega_{*}-10 \omega_{d}\right]$, which is stable in the present configuration.

The dispersion relation for the pure drift-wave mode can be obtained by setting $\omega_{d}=0$. In particular, in the case of $\nu=0$ and $m_{e} / m_{i}=0$, one obtains the usual stable drift wave, 
$\gamma=i \omega_{*}(1.71 \eta+1) /\left(1+2.95 k_{y}^{2}\right)$. On the other hand, resistivity and finite electron mass can destabilize drift waves. The fastest growing instability has $k_{y} \sim 1$, finite $k_{\|}$, and a growth rate $\gamma \sim \omega_{*}$. We note that if one assumes an isothermal equation of state, the dispersion relation for the drift waves in Ref. [14] is found. It is expected that the drift wave dominates over the interchange mode in the presence of steep gradients, since the ratio of their growth rates scales as $1 / \sqrt{L_{n}}$. Finally, neglecting ion parallel dynamics, the dispersion relation in Ref. [5] is obtained.

The linear growth rate of the most unstable mode, as predicted by Eq. (11), is shown in Fig. 1. The drift wave dominates at gradients steeper than $L_{n} / R \simeq 0.04$. The peak growth rate of the drift wave occurs at $k_{y} \simeq 0.5$, and at a $k_{\|}$that increases with $\nu$. The growth rate of the drift wave decreases when the resistivity is enhanced. At gradients weaker than $L_{n} / R \simeq 0.04$, the interchange mode becomes dominant. In this case, the peak growth rate is observed for $k_{y} \rightarrow 0$ and $k_{\|} \rightarrow 0$. The interchange instability persists at finite values of $k_{\|}$, destabilized by finite electron mass and resistivity.

We now consider the complete linearized system (6-10), focusing on the typical parameters of the nonlinear simulations discussed below. Figures 2 and 3 examine the linear instability in a system with finite size in the $z$ direction. For simplicity, we consider an equilibrium velocity profile $\bar{V}_{\|}(z)=2 \sqrt{T_{e 00}} z / L_{c}$. In comparison to the homogeneous case, the presence of the sheaths at $z= \pm L_{c} / 2$ constrains the allowed $k_{\|}$and introduces the boundary conditions for $\tilde{V}_{\| e}$ and $\tilde{V}_{\| i}$. Moreover, the combination of the sheath terms and the temperature gradient can introduce an instability into the system (see, e.g., Ref. [15]). As discussed in Ref. [4], for our parameters, this instability seems to be overpowered by the interchange mode.

In Fig. 2, the linear growth rate of the fastest growing mode is shown as a function of $k_{y}$, for different values of $L_{n}, \nu$, and $\sigma$. At steep gradients, one observes that the peak growth rate occurs at $k_{y} \sim 0.5$; the growth rate weakly decreases with the resistivity; moreover, it is essentially independent of $\sigma$. As confirmed by the eigenvalues plotted in Fig. 3, and according to the results obtained for homogeneous systems, for steep gradients, this dominant instability corresponds to a drift wave. At weaker gradients, the eigenfunction in Fig. 3 shows that the interchange instability dominates. We note that in the presence of sheath effects, the linear grow rate of the interchange instability is reduced at small value of $k_{y}$, as noted in the analysis of the two-dimensional model $[6,16]$. By balancing the 

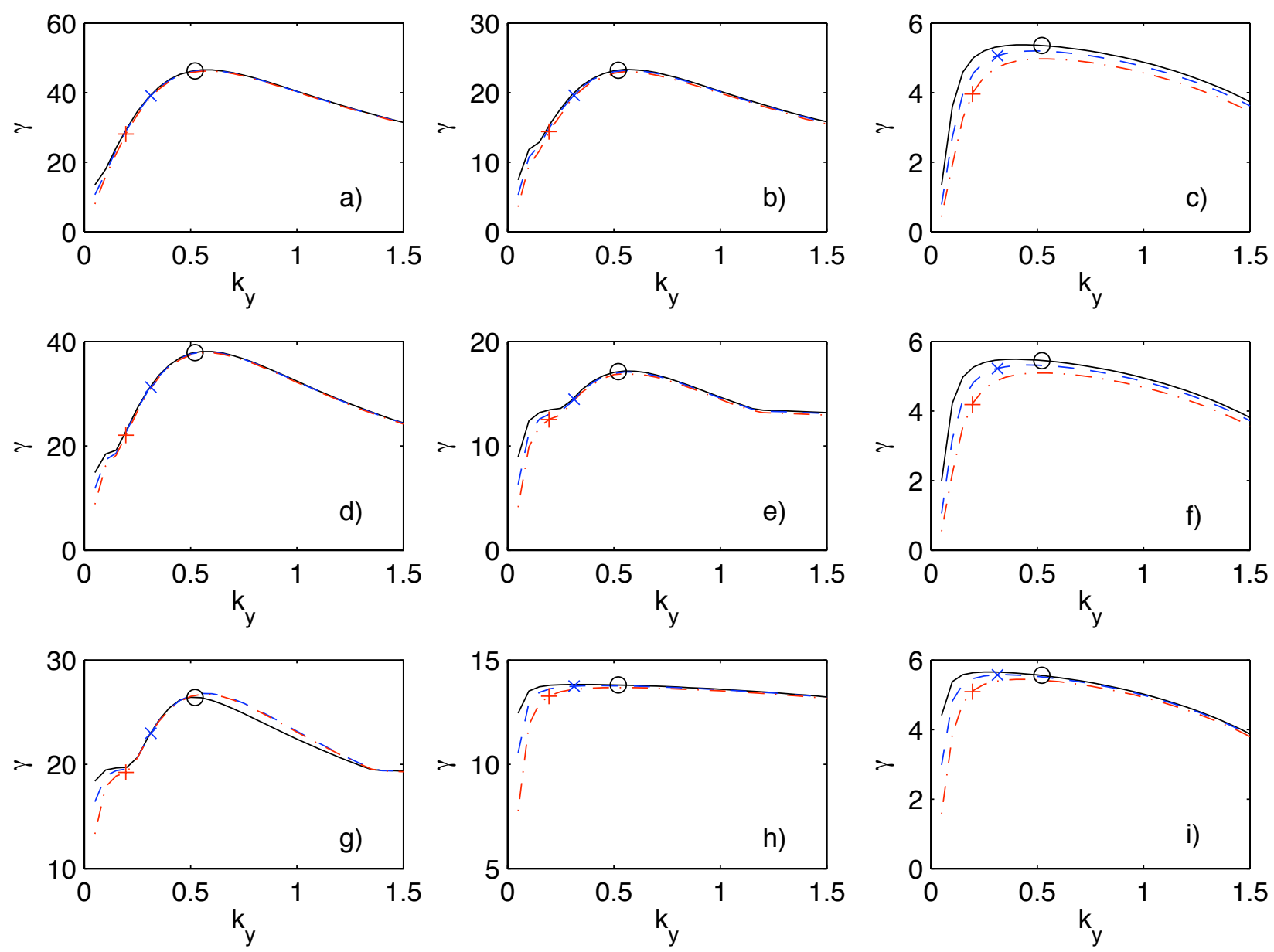

FIG. 2: (Color online) Linear growth rate of the most unstable mode evaluated from the linear instability associated to system (6-10). $L_{n} / R=0.01$ (left panels: a, d, and g), $L_{n} / R=0.02$ (middle panels: b, e, and $\mathrm{h}$ ), and $L_{n} / R=0.1$ (right panels: c, f, and i) is considered; $\nu=0.001$ (upper panels: a-c), $\nu=1$ (middle panels: d-f), and $\nu=0.1$ (lower panels: g-i). Solid black line corresponds to $\sigma=0.03$, dashed blue line to $\sigma=0.05$, dash-dotted red line to $\sigma=0.08$; the values of the growth rates for $k=k_{\Delta}$ are indicated with a black circle $(\sigma=0.03)$, blue 'x' mark $(\sigma=0.05)$, and red ' + ' $\operatorname{mark}(\sigma=0.08)$. It is $\eta=1, m_{i} / m_{e}=400, \Lambda=3$, and $L_{v}=64$.

term $\gamma k_{y}^{2} \tilde{\phi}$ and $d_{z} \tilde{j}_{\|} / n_{00}$ in Eq. (7), one notices that the cut-off of the growth rate occurs for $k_{y}^{2} \sim 1 /\left(\sqrt{T_{e 00}} L_{z} \gamma\right)$. As expected from an instability driven by curvature and pressure gradient, the dependence on the resistivity is weak; the linear growth rate of the interchange is instead reduced by $\sigma$.

Because of the finite extension of the system along the $y$ direction, $k_{y}$ is discretized to 

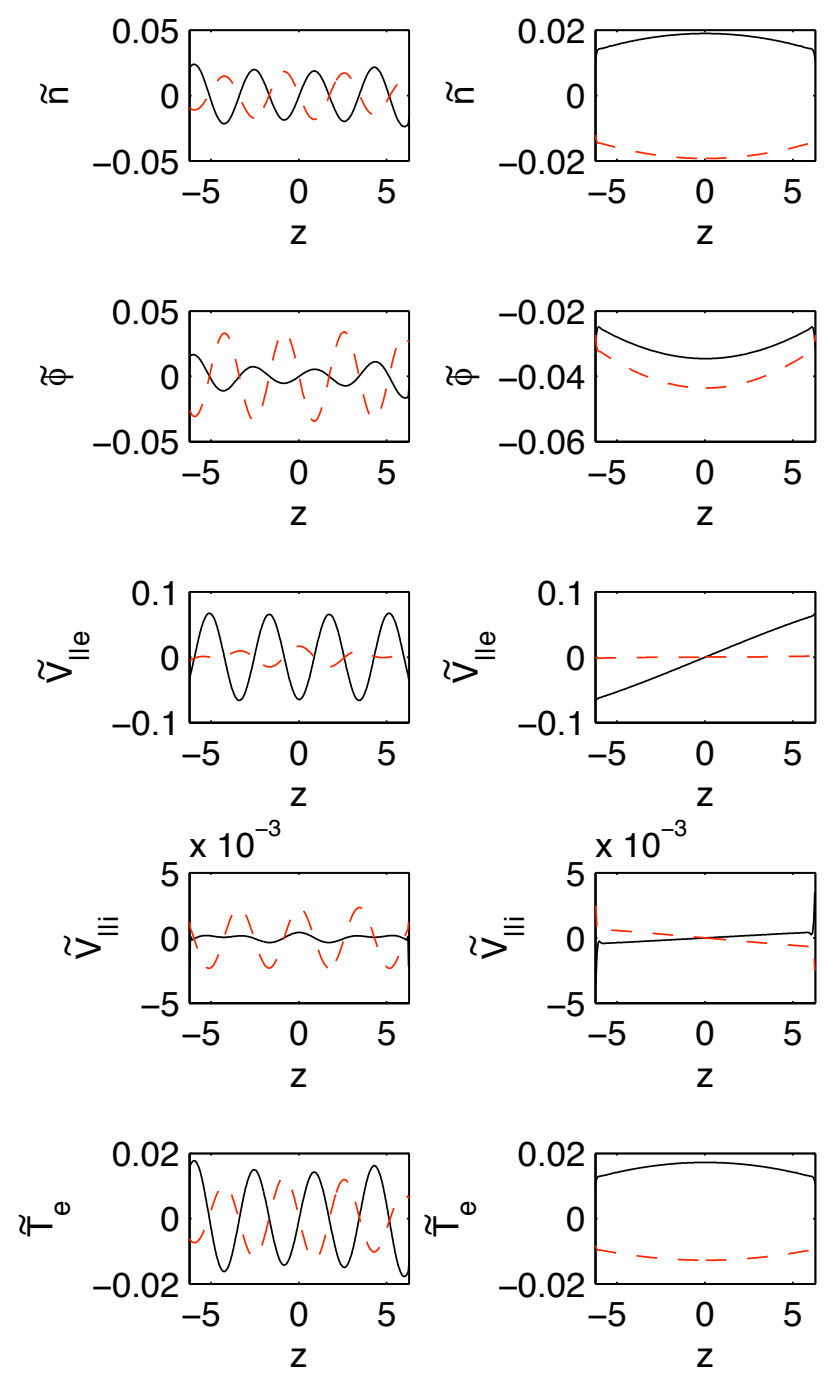

FIG. 3: (Color online) Real part (black solid line) and imaginary part (red dashed line) of the fastest growing eigenfunction of system (6-10) for $L_{n} / R=0.01$ (left panels) and $L_{n} / R=0.1$ (right panels). It is $\eta=1, \sigma=0.08, \nu=1, m_{i} / m_{e}=400, L_{v}=64$, and $\Lambda=3$.

multiples of $k_{\Delta}=2 \pi / \Delta$. In Fig. 2, the growth rate corresponding to $k_{y}=k_{\Delta}$ is indicated. In the cases where the interchange instability dominates, one can observe that typically the fastest growing mode occurs at $k_{y}=k_{\Delta}$. The only exception is for $\sigma=0.08$ and large $\nu$. In this case, the fastest growing instability has $k_{y}>k_{\Delta}$. 


\section{NON-LINEAR REGIMES}

We start the simulations from random noise; particles and heat are then introduced by the sources in a radially localized region, steepening the $n$ and $T_{e}$ profiles, until the interchange and the drift-wave instabilities are triggered. These instabilities drive turbulence that causes plasma transport in the radial direction. After a transient, a quasi-steady state is established in which plasma, introduced by the plasma sources, is transported in the perpendicular direction and is eventually lost from the system at the sheath edges. We focus here on this quasi-stationary state.

Typical equilibrium profiles obtained from the solution of Eqs. (1-5) are shown in Fig. 4 and typical snapshots of the turbulent dynamics are presented in Fig. 5. [We denote equilibrium profiles with the overline and fluctuations with the tilde, e.g. $n(x, y, z, t)=$ $\tilde{n}(x, y, z, t)+\bar{n}(x, z)]$. Three turbulence regimes are observed. (i) For small $\nu$, small values of $S_{0}$ and large values of $\sigma$, turbulence snapshots show the presence of intermittent transport events that have a flute character. In this turbulent regime, shear flow plays a minor role and plasma is transported from the source region to the low-field side of the machine by the interchange instability, while it is progressively lost at the plasma sheaths. This results in smooth exponentially decaying plasma radial profiles. The $\bar{n}, \bar{\phi}$, and $\bar{T}_{e}$ profiles show a weak parabolic dependence on the parallel direction, while $\bar{V}_{\| e}$ and $\bar{V}_{\| i}$ linearly depend on the $z$ coordinate. As expected from the quasi-neutrality conditions, one observes that $\bar{V}_{\| e} \simeq \bar{V}_{\| i}$ at $z= \pm L_{c} / 2$; this implies that $\bar{\phi} \simeq \Lambda \bar{T}_{e}$ at $z= \pm L_{c} / 2$. One also notes that, at least approximately, $\bar{\phi} \simeq \Lambda \bar{T}_{e}$ throughout the domain. Following the notation of Ref. [6] we denote this turbulence regime as low confinement mode, L-mode. (ii) For small values of $\sigma$, large $S_{0}$, and small $\nu$, the $\bar{\phi}$ and $\bar{T}_{e}$ profiles weakly depend on $z$ and $\bar{\phi} \simeq \Lambda \bar{T}_{e}$ in the whole domain, as in the L-mode. However, shear flow is important, the plasma profiles are steeper than in the L-mode, and intermittent transport events are not visible. We denote this turbulent regime as the high confinement mode, H-mode, since it is similar to the high confinement mode first described in Ref. [6]. (iii) For large values of $\nu$, the $\bar{n}$ and $\bar{T}_{e}$ profiles show steeper radial gradients with respect to the L-mode. The relation $\bar{\phi} \simeq \Lambda \bar{T}_{e}$ is only satisfied at $z= \pm L_{c} / 2$ and $\bar{\phi}$ decouples from $\bar{T}_{e}$ at $|z|<L_{c} / 2$, assuming a negative value and creating a strong shear flow that limits the perpendicular transport. We denote this high confinement regime driven by resistivity, inherently three-dimensional and not observed 

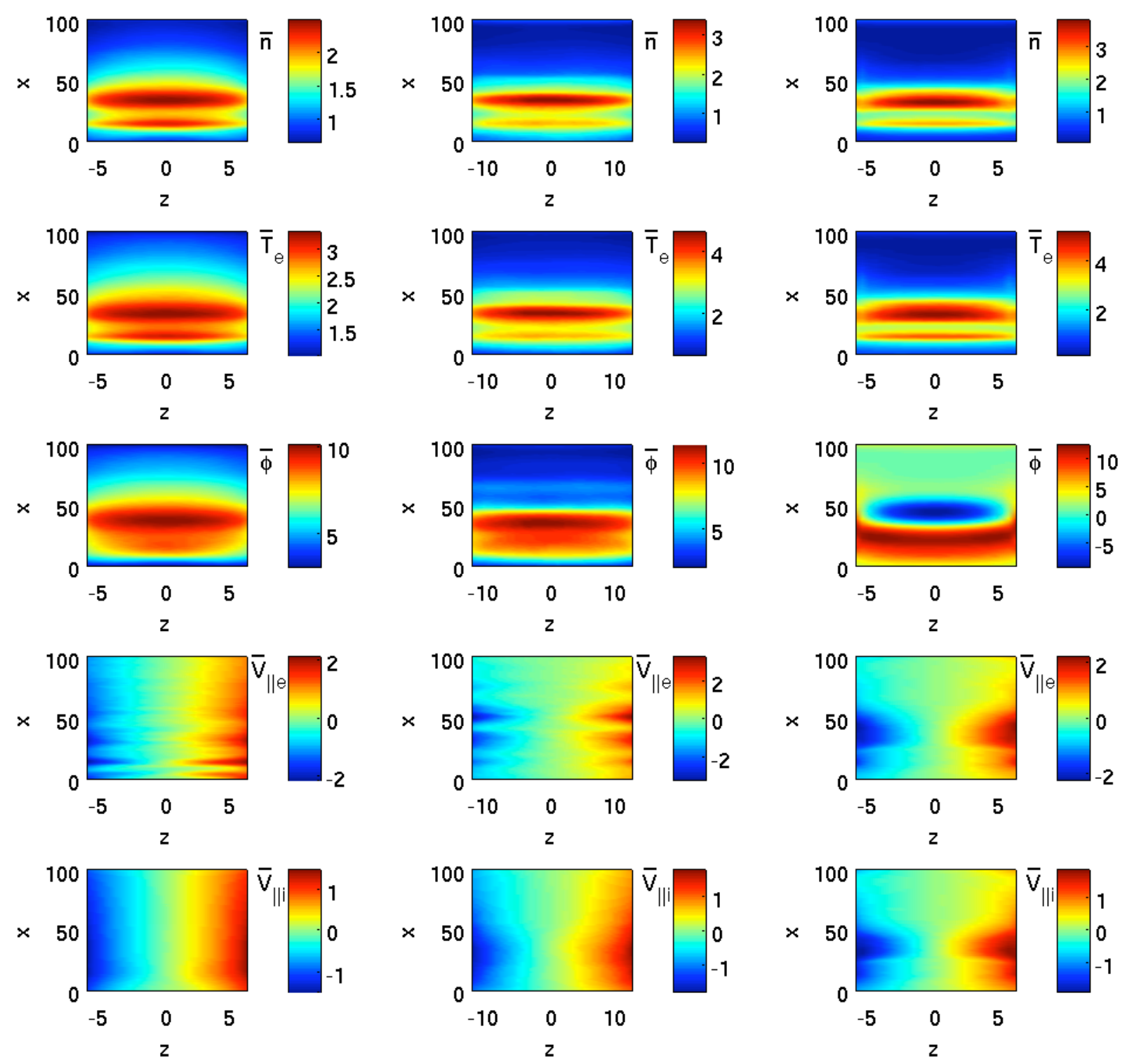

FIG. 4: (Color online) Time averaged profiles, $\bar{n}, \bar{\phi}, \overline{T_{e}}, \bar{V}_{\| e}$, and $\bar{V}_{\| i} \cdot \sigma=0.08, S_{0}=2$, and $\nu=0.0001$ (left panels), corresponding to the L-mode; $\sigma=0.04 n=0.1$, and $S=1$ (central panels), corresponding to the H-mode; $\sigma=0.08, S_{0}=1$, and $\nu=10$ (right panels), corresponding to the $\nu \mathrm{H}$-mode.

previously in two-dimensional simulations, as $\nu \mathrm{H}$-mode.

According to expectations from the linear theory, in the L-mode in which plasma gradients are weak, the typical snapshots of Fig. 5 show that turbulence has an interchange character, with $k_{\|} \simeq 0$ and $k_{y} \simeq k_{\Delta}$; we note that the interchange character of turbulence is observed for 

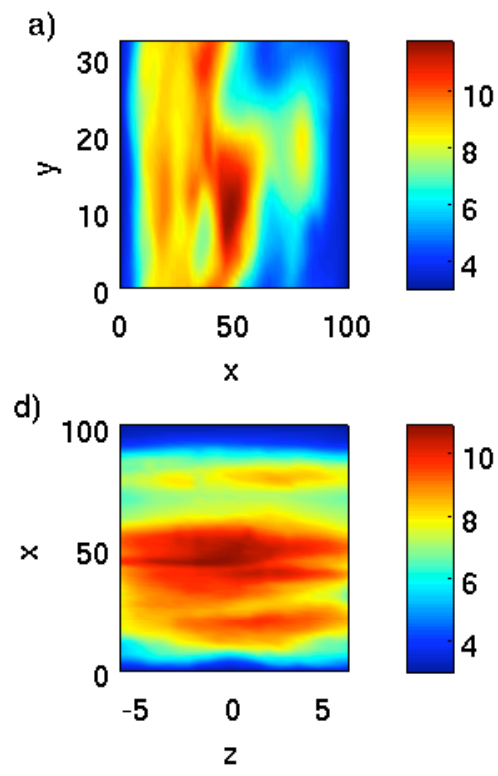

b)

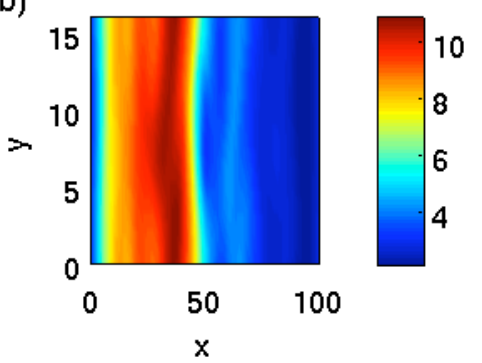

e)

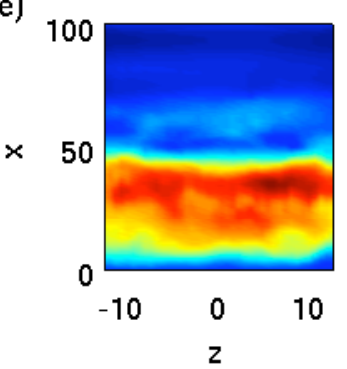

c)

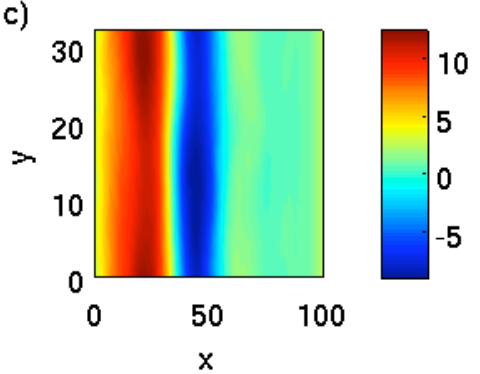

f)

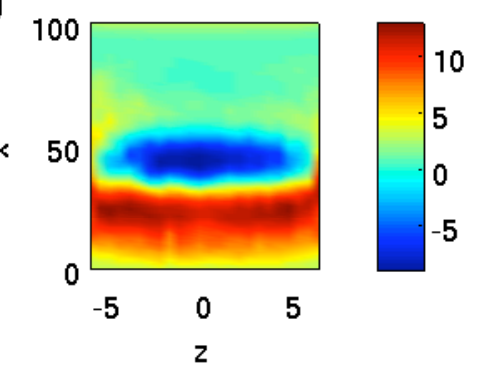

FIG. 5: (Color online) Typical $\phi$ snapshots in the $z=0$ plane (upper panels: a-c) and at $y=L_{y} / 2$ (lower panels: d-f). $\sigma=0.08, S_{0}=2$, and $\nu=0.0001$ (left panels: a and d); $\sigma=0.04 n=0.1$, and $S=1$ (central panels: b and e); $\sigma=0.08, S_{0}=1$, and $\nu=10$ (right panels: c and f).

all the L-mode simulations considered in the present study. In the $\mathrm{H}$ - and $\nu \mathrm{H}$-modes, when gradients becomes steep, and the linear theory shows that drift-wave importance increases with respect to the interchange instability, a strong shear flow arises that quenches the turbulence.

We first focus on the L-mode, where the plasma profiles can be evaluated analytically. We begin with the study of the peak value of density and temperature, $n_{\max }(z)$ and $T_{e, \max }(z)$, approximately located at the peak source location, $x=x_{S}\left(x_{S} \simeq 36\right.$ in the present simulation). We note that the density and temperature perpendicular transport, $\Gamma_{n}=R\left\langle\overline{\tilde{n} \partial_{y} \tilde{\phi}}\right\rangle_{y}$ and $\Gamma_{T}=R\left\langle\overline{\tilde{T}_{e} \partial_{y} \tilde{\phi}}\right\rangle_{y}$, vanish at $x=x_{S}$. By Taylor expanding $\bar{V}_{\| e}, \bar{n}$, and $\bar{T}_{e}$ at $z=0$, one obtains $n_{\max }(z)=\bar{n}\left(x_{s}, 0\right)\left(1+\zeta z^{2} / 3\right)$ and $T_{e, \max }(z)=\bar{T}_{e}\left(x_{S}, 0\right)\left(1+\zeta z^{2} / 4\right)$. By inserting these Taylor expansions into Eq. (5), and approximating $\bar{V}_{\| i}\left(x_{S}, z\right) \simeq 2 \sqrt{T_{e, \max }\left( \pm L_{c} / 2\right)} z / L_{c}$, it is possible to deduce $\zeta=48 / 17$. It follows

$$
n_{\max }(z)=n\left(x_{S}, 0\right)\left(1-\frac{16 z^{2}}{17 L_{c}^{2}}\right), T_{e, \max }(z)=T\left(x_{S}, 0\right)\left(1-\frac{12 z^{2}}{17 L_{c}^{2}}\right)
$$

We note that the simulation results also show that the ratio between the den- 

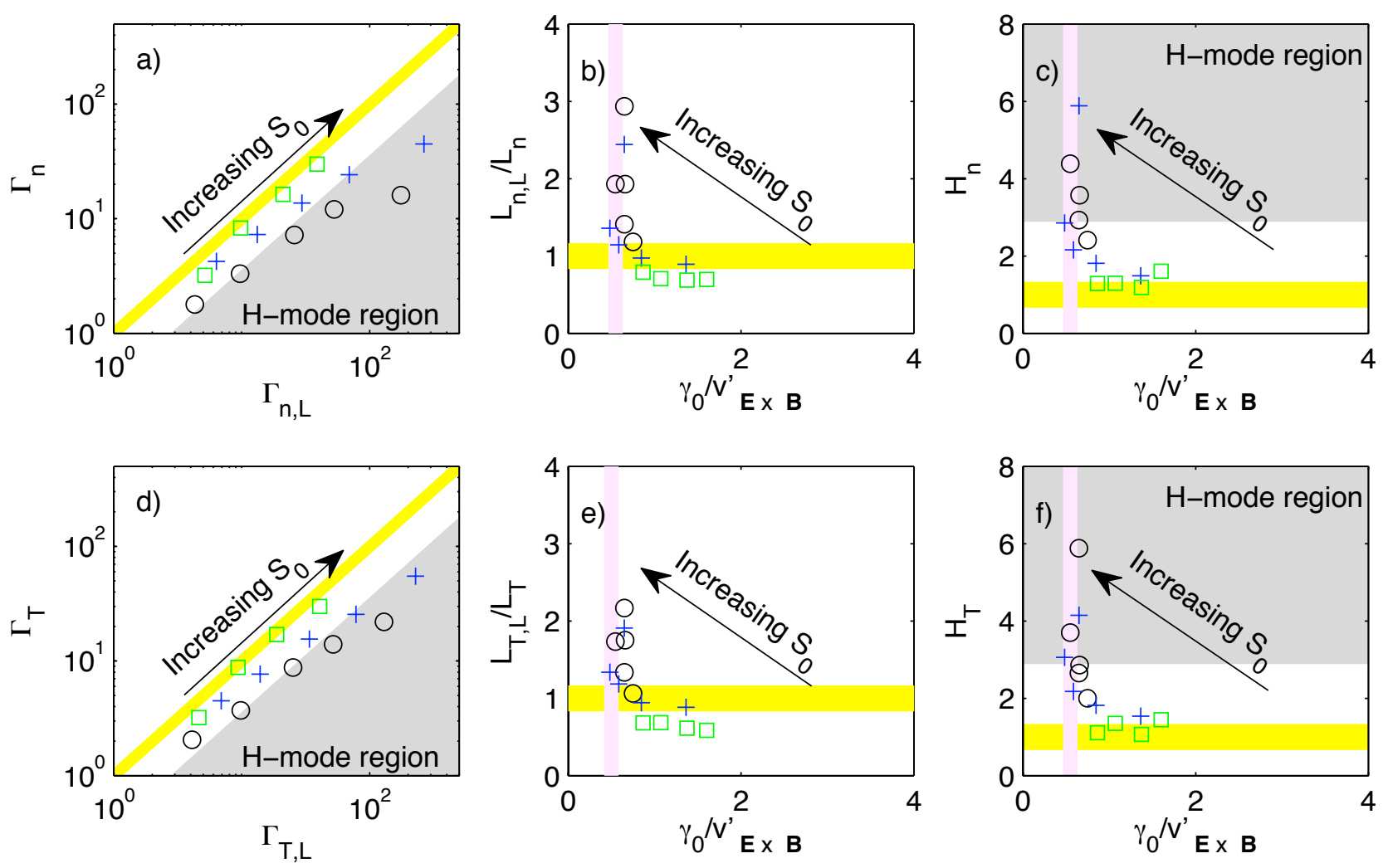

FIG. 6: (Color online) $\Gamma_{n}$ vs. $\Gamma_{n, L}, L_{n, L} / L_{n}$ vs. $\gamma / v_{\mathbf{E} \times \mathbf{B}}^{\prime}$, and $H_{n}$ vs. $\gamma / v_{\mathbf{E} \times \mathbf{B}}^{\prime}$ (upper panels: a-c) and $\Gamma_{T}$ vs. $\Gamma_{T, L}, L_{T, L} / L_{T}$ vs. $\gamma / v_{\mathbf{E} \times \mathbf{B}}^{\prime}$, and $H_{T}$ vs. $\gamma / v_{\mathbf{E} \times \mathbf{B}}^{\prime}$ (lower panels: d-f). It is $\sigma=0.04$ (black circles), $\sigma=0.05$ (blue crosses), and $\sigma=0.08$ (green squares). $\nu=0.01$.

sity at $z=0$ and $z= \pm L_{c} / 2$ is approximately constant for $x \geq x_{S}$, i.e. $\bar{n}\left(x, \pm L_{c} / 2\right) / \bar{n}(x, 0) \simeq n_{\max }\left( \pm L_{c} / 2\right) / n_{\max }(0) \simeq 13 / 17$. Similarly, one has $\bar{T}_{e}\left(x, \pm L_{c} / 2\right) / \bar{T}_{e}(x, 0) \simeq T_{e, \max }\left( \pm L_{c} / 2\right) / T_{e, \max }(0) \simeq 14 / 17$, for $x \geq x_{S}$.

The $z$-dependence of $\bar{\phi}$ can be deduced from Eq. (4). In the analysis of the L-mode, we consider the $\nu \rightarrow 0$ limit; i.e., the leading order terms in Eq. (4) are $\bar{n} \partial_{z} \bar{\phi} \simeq \bar{T}_{e} \partial_{z} \bar{n}+$ $1.71 \bar{n} \partial_{z} \bar{T}_{e}$. Using Eqs. (18), one obtains

$$
\bar{\phi}(x, z)=\bar{T}_{e}(x, 0)\left(\frac{14 \Lambda}{17}+0.54-2.15 z^{2} / L_{c}^{2}\right)
$$

for $x>x_{S}$, by imposing that $\bar{\phi}\left(x, \pm L_{c} / 2\right)=\Lambda \bar{T}_{e}\left(x, \pm L_{c} / 2\right)$. For later use, we note the $z$ scale length of the potential at $z=L_{c} / 2$ for $\nu \rightarrow 0$ is given by $L_{z \phi, 0}=\bar{\phi} /\left.\partial_{z} \bar{\phi}\right|_{z=L_{c} / 2} \simeq-\Lambda L_{c} / 2.61$ for $x>x_{S}$.

We now estimate the $\bar{T}_{e}$ and $\bar{n}$ radial profiles. At $z=0$, since from Eq. (18), $\bar{V}_{\| e} \simeq$ 
$\sqrt{56 / 17} \bar{T}_{e}(x, 0) z / L_{c}$ for $x>x_{S}$, the $y$ and time average of Eqs. (1) and (3) lead to

$$
\frac{\partial \Gamma_{n}}{\partial x} \simeq S_{n}-2 \sigma \sqrt{\frac{14}{17}} \bar{n} \bar{T}_{e}^{1 / 2}, \frac{\partial \Gamma_{T}}{\partial x} \simeq S_{T}-\frac{4}{3} \sigma \sqrt{\frac{14}{17}} \bar{T}_{e}^{3 / 2}
$$

In the L-mode regime, it is possible to analytically estimate the fluxes $\Gamma_{n}$ and $\Gamma_{T}$. Since in the L-mode transport is driven by the interchange mode, and we focus on the regime where the dominant mode has $k_{y}=k_{\Delta}$, the nonlocal quasi-linear theory developed for the twodimensional model in Ref. [6] is valid. The estimate shows that in the absence of shear flow and far from marginal stability, transport can be approximated as $\Gamma_{n}=\Gamma_{n, L}$ and $\Gamma_{T}=\Gamma_{T, L}$, with

$$
\Gamma_{n, L}=\alpha_{n} \frac{\bar{n}\left(2 R L_{p} \bar{T}_{e}\right)^{1 / 2}}{L_{n} k_{\Delta}}, \Gamma_{T, L}=\alpha_{T} \frac{\left(2 R L_{p} \bar{T}_{e}^{3}\right)^{1 / 2}}{L_{T} k_{\Delta}}
$$

and $\alpha_{n} \simeq \alpha_{T} \simeq 0.4$. Introducing Eqs. (21) into Eqs. (20) and considering the source free region, one finds that $\bar{n}(x, 0)=n_{\max }(0) \exp \left[\left(x-x_{S}\right) / L_{n, L}\right]$ and $\bar{T}_{e}(x, 0)=T_{e, \max }(0) \exp [(x-$ $\left.\left.x_{S}\right) / L_{T, L}\right]$ are solutions of Eq. (20) with $L_{n, L}=0.47\left(L_{v} \sqrt{R}\right)^{2 / 3}$ and $L_{T, L}=0.60\left(L_{v} \sqrt{R}\right)^{2 / 3}$.

In Figs. 6 and 7 we study the agreement between the L-mode estimate and the simulation results. Figure 6 focuses on the small $\nu$ case. According to the findings in Ref. [6], one observes that transport is reduced and gradients become steeper with respect to the Lestimate for small values of $\sigma$ and large values of $S_{0}$. Figures $6 \mathrm{~b}$ and $6 \mathrm{e}$ shows that deviations from the L-mode are due to shear flow, $v_{\mathbf{E} \times \mathbf{B}}^{\prime}=R \partial_{x}^{2} \phi$, and become important for $\gamma / v_{\mathbf{E} \times \mathbf{B}}^{\prime} \simeq$ 0.5. We introduce the $H$ parameters, $H_{n}=\Gamma_{n, L} / \Gamma_{n}$ and $H_{T}=\Gamma_{T, L} / \Gamma_{T}$, and define the H-mode simulations as those for which $H_{n}>3$ or $H_{T}>3$ (both criteria lead essentially to the same results). From Figs. 6c and 6f, one observes that the transition to the H-mode occurs for $\gamma / v_{\mathbf{E} \times \mathbf{B}}^{\prime} \simeq 0.5$. (We point out that, in the L-mode, the small deviation of the simulated $L_{n}$ and $L_{T}$ from the analytical estimate given here is due to the finite domain extension along the $x$ direction.) The dependence on $\nu$ is studied in Fig. 7. Figure 7 shows that an enhancement of $\nu$ leads to a reduction of transport, a steepening of the plasma profiles, and an increase of $v_{\mathbf{E} \times \mathbf{B}}^{\prime}$. The transition from the L-mode to the $\nu \mathrm{H}$-mode also occurs for $\gamma / v_{\mathbf{E} \times \mathbf{B}}^{\prime} \simeq 0.5$.

In conclusion, the present three-dimensional simulations, besides supporting the findings in Ref. [6] regarding the existence of an L-H transition, show that a transition to a regime where turbulence is suppressed by shear flow occurs when $\nu$ is increased. It is thus possible to access a quenched turbulence regime by increasing the value of $\nu$ and $S_{0}$, and decreasing 

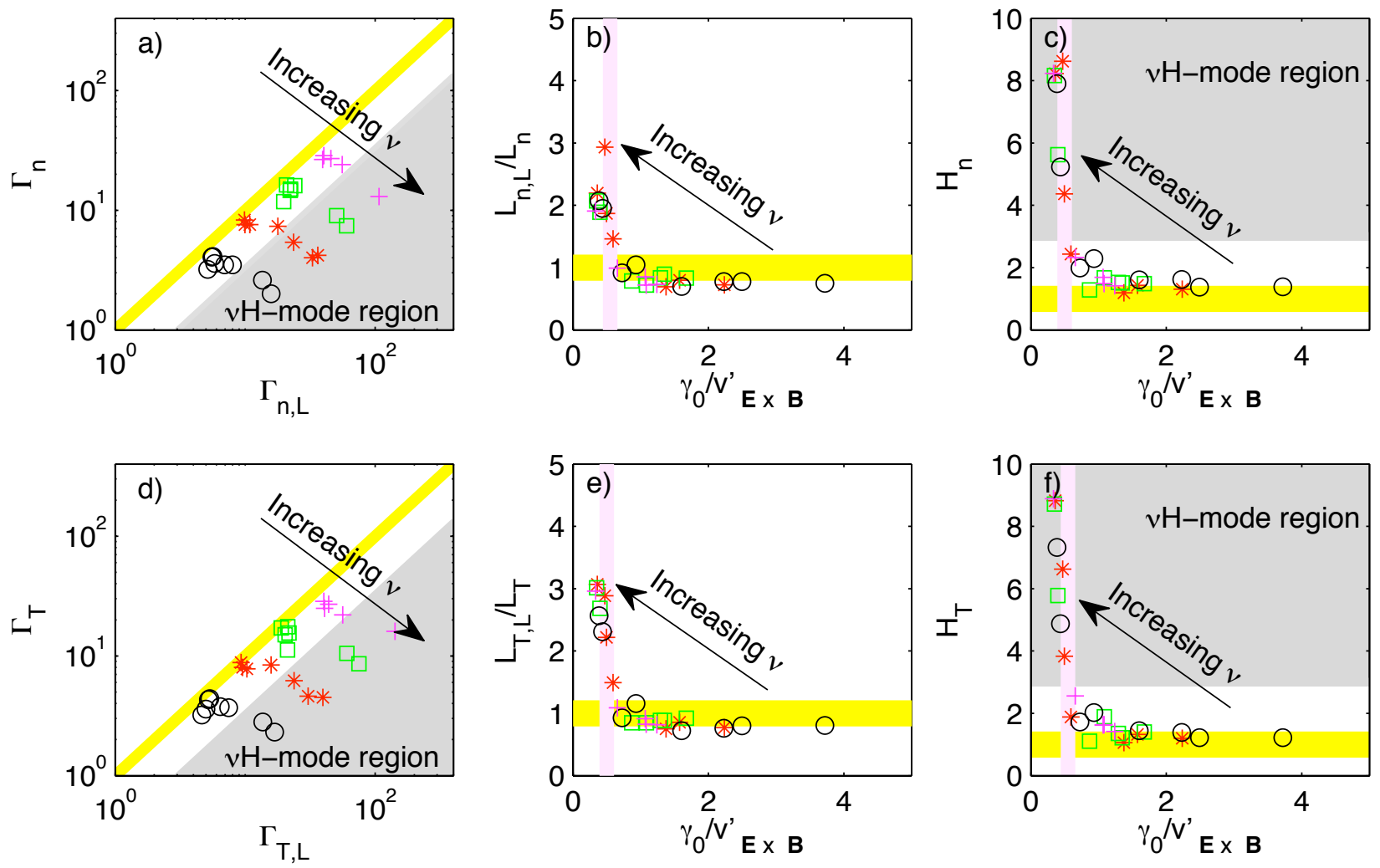

FIG. 7: (Color online) $\Gamma_{n}$ vs. $\Gamma_{n, L}, L_{n, L} / L_{n}$ vs. $\gamma / v_{\mathbf{E} \times \mathbf{B}}^{\prime}$, and $H_{n}$ vs. $\gamma / v_{\mathbf{E} \times \mathbf{B}}^{\prime}$ (upper panels: a-c) and $\Gamma_{T}$ vs. $\Gamma_{T, L}, L_{T, L} / L_{T}$ vs. $\gamma / v_{\mathbf{E} \times \mathbf{B}}^{\prime}$, and $H_{T}$ vs. $\gamma / v_{\mathbf{E} \times \mathbf{B}}^{\prime}$ (lower panes: d-f). It is $S_{0}=0.5$ (black circles), $S_{0}=1$ (red stars), $S_{0}=2$ (green squares), $S_{0}=4$ (purple '+' marks). $\sigma=0.08$. Different values of $\nu$ are considered.

$\sigma$

\section{THRESHOLD CONDITION TO A QUENCHED TURBULENCE REGIME}

We now estimate the threshold condition to a quenched turbulence regime, occurring for $\gamma / v_{\mathbf{E} \times \mathbf{B}}^{\prime} \simeq 0.5$. We first consider the $\nu \rightarrow 0$ case. In this limit, as in the two-dimensional simulations, shear flow is mostly due to a sheared temperature profiles, i.e. $\partial_{x}^{2} \bar{\phi} \simeq \Lambda \partial_{x}^{2} \bar{T}_{e}$, and it is limited by a Kelvin-Helmholtz $(\mathrm{KH})$ instability. The KH stability condition imposes $k_{y} L_{\phi}>\kappa(\kappa \simeq 2$ for a Bickley jet profile [17]). Since the most stringent criterion for stability 
occurs for $k_{y}=k_{\Delta}$, one deduces

$$
\frac{\partial^{2} \bar{\phi}}{\partial x^{2}} \simeq \chi \frac{\Lambda \bar{T}_{e, \max }}{\Delta^{2}}
$$

in good agreement with simulation results for $\chi \simeq 2.5$.

In the $\nu$ H-mode, $\bar{\phi}$ decouples from $\bar{T}_{e}$ away from the sheaths, and shear flow is due to a strong radial dependence of $\bar{\phi}$ resulting from the fact that in the source region $\bar{\phi} \simeq \Lambda \bar{T}_{e}$, while at the right of the plasma source there is a potential cavity where $\bar{\phi} \ll \Lambda \bar{T}_{e}$. Analytical estimates can be carried out in this regime, in order to evaluate the depth of the potential cavity. The quantity $x_{W}$ denotes the radial position where $\bar{\phi}$ assumes its minimum value, $\bar{\phi}\left(x_{W}, 0\right)$. We note that $x \simeq x_{W}$ is also the location where $\bar{j}_{\|}$is largest. Numerical tests show that the radial distance $\delta=x_{S}-x_{W}$ between the maximum and minimum value of $\bar{\phi}$ depends mainly on the geometrical parameters of the SMT, i.e. $\delta \simeq \delta\left(L_{v}, R\right) \simeq 0.35 L_{T, L}$ for the present simulations.

We first consider the physics at $x=x_{W}$. For $\nu \rightarrow \infty$, the leading order terms in Eq. (4) give

$$
\frac{\partial \bar{\phi}}{\partial z}+\nu \bar{j}_{\|} \simeq 0
$$

A second relation between $\bar{\phi}$ and $\bar{j}_{\|}$is provided by the time and $y$ average of Eq. (2),

$$
R \frac{\partial^{2}\left\langle\tau_{x y}\right\rangle_{y}}{\partial x^{2}}+\overline{\tilde{V}_{\| i} \frac{\partial^{3} \tilde{\phi}}{\partial z \partial x^{2}}}+\bar{V}_{\| i} \frac{\partial^{3} \bar{\phi}}{\partial z \partial x^{2}} \simeq \frac{1}{\bar{n}} \frac{\partial \bar{j}_{\|}}{\partial z}
$$

where $\tau_{x y}=\overline{\partial_{x} \tilde{\phi} \partial_{y} \tilde{\phi}}$ is the Reynold stress term. For $z \rightarrow 0$, the leading order terms in Eq. (24) are

$$
R \frac{\partial^{2}\left\langle\tau_{x y}\right\rangle_{y}}{\partial x^{2}}+\overline{\tilde{V}_{\| i} \frac{\partial^{3} \tilde{\phi}}{\partial x^{2} \partial z}} \simeq \frac{1}{\bar{n}} \frac{\partial \bar{j}_{\|}}{\partial z}
$$

since $\bar{V}_{\| i} \rightarrow 0$ for $z \rightarrow 0$; on the other hand, for $z \rightarrow \pm L_{c} / 2$, the leading order terms of Eq. (24) are

$$
\bar{V}_{\| i} \frac{\partial^{3} \bar{\phi}}{\partial z \partial x^{2}} \simeq \frac{1}{\bar{n}} \frac{\partial \bar{j}_{\|}}{\partial z} .
$$

We focus on the latter limit, Eq. (26), which is more easily treated. For $z \rightarrow L_{c} / 2$ and $\nu \rightarrow \infty$, Eqs. (23) and (26) lead to $-\nu \bar{n} \bar{V}_{\| i} \partial_{x}^{2} \bar{j}_{\|} \simeq \partial_{z} \bar{j}_{\|}$; it is thus possible to estimate the $\nu \rightarrow \infty z$-scale length of $j_{\|}, L_{z j, \infty}$, i.e.

$$
L_{z j, \infty}=\left.\frac{\bar{j}_{\|}}{\partial_{z} \bar{j}_{\|}}\right|_{z=L_{c} / 2, x=x_{W}} \simeq-\frac{\bar{j}_{\|}}{\nu \bar{n} \bar{V}_{\| i} \partial_{x}^{2} \bar{j}_{\|}}
$$


Since the value of $\partial_{x}^{2} \bar{\phi}$ is limited by the KH stability condition and thus $\partial_{x}^{2} \bar{\phi} \propto 1 / \Delta^{2}$, from Eq. (26), one expects that the value of $\bar{j}_{\|}$is also tied to the $\mathrm{KH}$ threshold. In particular, simulations show that $\partial_{x}^{2} \bar{j}_{\|} \simeq-\chi_{j} \bar{j}_{\|} / \Delta^{2}$, with $\chi_{j} \simeq 22$. Introducing this estimate into Eq. (27), one obtains $L_{z j, \infty}=\Delta^{2} /\left(\chi_{j} \nu \bar{n} \bar{V}_{\| i}\right)=\Delta^{2} /\left(\chi_{j} \nu \bar{n} \sqrt{\bar{T}_{e}}\right)$. It is observed that the scale length of the potential in the $\nu \rightarrow \infty$ limit, $L_{z \phi, \infty}$, is proportional to $L_{z j, \infty}$, resulting in

$$
L_{z \phi, \infty}=\frac{\varsigma \Delta^{2}}{\chi_{j} \nu \bar{n}\left(x_{W}, \pm L_{c} / 2\right) \sqrt{\bar{T}_{e}\left(x_{W}, \pm L_{c} / 2\right)}}
$$

with the proportionality constant $\varsigma \simeq 0.2$.

In general, the scale length of $\bar{\phi}$ should gradually pass from the $\nu \rightarrow 0$ to the $\nu \rightarrow \infty$ limit with increasing resistivity. We can thus approximate $\partial_{x} \phi /\left.\phi\right|_{z= \pm L_{c} / 2, x=x_{W}}=1 / L_{z \phi}=$ $1 / L_{z \phi, 0}+1 / L_{z \phi, \infty}$. The impact of plasma resistivity on the plasma profile becomes relevant when $L_{z \phi, \infty}<L_{c} / 2$, i.e. $\nu>2 \varsigma \Delta^{2} /\left(\chi_{j} \bar{n} \sqrt{\bar{T}_{e}} L_{c}\right)$.

Let us now consider the physics in the source region, $x=x_{S}$. Fluctuations are small in this region, since density and temperature gradients are weak. Thus, the Reynold stress and the cross correlation term $\overline{\tilde{V}_{\| i} \partial_{z} \partial_{x}^{2} \tilde{\phi}}$ in Eq. (23) vanish and Eq. (26) is valid for all z. By Taylor expanding Eq. (26) with respect to $z$, one observes that the leading order term of $\bar{j}_{\|}$vanishes; simulations confirm that $\bar{j}_{\|}$is negligible at $x=x_{S}$. It follows that the $\nu \rightarrow 0$ leading order terms balance of Eq. (4) is valid and, since the $z$-dependence of $\phi_{\max }$ is given by Eq. (19), $\bar{\phi}$ and $\bar{T}_{e}$ remain coupled for all $z$ at $x=x_{S}$.

Since we are interested in evaluating the threshold condition to a quenched turbulent regime due to resistivity, we focus on the $\nu \rightarrow \infty$ case, and in this limit we now estimate $\bar{\phi}\left(x_{W}, 0\right)$. Simulations show that a polynomial expression represents reasonably well the $z$ dependence of $\bar{\phi}$, i.e. $\bar{\phi}\left(x_{W}, z\right)=\bar{\phi}\left(x_{W}, 0\right)+\left(2 z / L_{c}\right)^{\alpha}\left[\bar{\phi}\left(x_{W}, \pm L_{c} / 2\right)-\bar{\phi}\left(x_{W}, 0\right)\right]$ (with $\alpha \simeq 4)$. Thus, one has

$$
\bar{\phi}\left(x_{W}, 0\right)=\left(1-\frac{L_{c}}{2 \alpha L_{z \phi, \infty}}\right) \bar{\phi}\left(x_{W}, \pm L_{c} / 2\right)=\left(1-\frac{L_{c}}{2 \alpha L_{z \phi, \infty}}\right) \bar{\phi}_{\max }\left( \pm L_{c} / 2\right) \exp \left(-\frac{\delta}{L_{\phi}}\right)
$$

At $z=0$, on the low-field side, and in the region where shear flow is maximum, a good approximation for $\bar{\phi}$ is provided by

$$
\bar{\phi} \simeq \frac{1}{2}\left[\phi_{\max }(0)+\bar{\phi}\left(x_{W}, 0\right)\right]+\frac{1}{2}\left[\phi_{\max }\left(x_{S}\right)-\bar{\phi}\left(x_{W}, 0\right)\right] \cos \left(k_{x} x\right)
$$


with $k_{x} \simeq k_{\Delta}$ set by the KH stability threshold condition for a sinusoidal $\bar{\phi}$ profile. Thus, from Eqs. (29) and (30), one can evaluate

$$
\begin{aligned}
\frac{\partial^{2} \bar{\phi}}{\partial x^{2}} \simeq \frac{k_{\Delta}^{2}}{2}\left[\phi_{\max }(0)-\bar{\phi}\left(x_{W}, \pm L_{c} / 2\right)\right] & +\frac{k_{\Delta}^{2} L_{c}}{4 \alpha L_{z \phi, \infty}} \bar{\phi}\left(x_{W}, \pm L_{c} / 2\right) \\
& \simeq \frac{k_{\Delta}^{2} L_{c}}{4 \alpha L_{z \phi, \infty}} \bar{\phi}\left(x_{W}, \pm L_{c} / 2\right)
\end{aligned}
$$

since $L_{z \phi, \infty} \rightarrow 0$ for $\nu \rightarrow \infty$. Since the $n_{\max }(z)$ and $T_{e, \max }(z)$ estimates, Eq. (18), carried out in the L-mode analysis, are valid also in the presence of a large resistivity, from Eqs. (28) and (31) one obtains

$$
\frac{\partial^{2} \bar{\phi}}{\partial x^{2}} \simeq \frac{13 \sqrt{14}}{17^{3 / 2}} \frac{k_{\Delta}^{2} L_{c} \chi_{j} \nu \sqrt{T_{e, \max }(0)}}{4 \varsigma \alpha \Delta^{2}} n_{\max }(0) \phi_{\max }\left( \pm L_{c} / 2\right) \exp \left[-\delta\left(\frac{1}{L_{\phi}}+\frac{1}{L_{n}}+\frac{1}{2 L_{T}}\right)\right]
$$

The values of $T_{e, \max }(0)$ and $n_{\max }(0)$ can be estimated by integrating Eqs. (20) along $x$. One has

$$
T_{e, \max }^{3 / 2}(0)=\frac{\sqrt{17} \int S_{T} d x}{2 \sqrt{14} \sigma\left(2 \xi x_{S} / 3+4 L_{T} / 9\right)}
$$

and

$$
n_{\text {max }}(0)=\frac{\int S_{n} d x\left(2 \xi x_{S} / 3+4 L_{T} / 9\right)}{(2 \sigma \sqrt{14 / 17})^{2 / 3}\left(\int S_{T} d x\right)^{1 / 3}\left[\xi x_{S}+2 L_{n} L_{T} /\left(L_{n}+2 L_{T}\right)\right]}
$$

At the threshold, it is reasonable to approximate $L_{\phi} \sim L_{T} \sim L_{n} \sim \Delta$. Summing the shear flow estimates in both the $\nu \rightarrow 0$ and the $\nu \rightarrow \infty$ limits, Eqs. (22) and (32), we approximate $\partial_{x}^{2} \bar{\phi} \simeq \chi \phi_{\max }\left( \pm L_{c} / 2\right) / \Delta^{2}+13 \sqrt{14} \phi_{\max }\left( \pm L_{c} / 2\right) k_{\Delta}^{2} L_{c} \chi_{j} \nu n_{\max }(0) \sqrt{T_{e, \max }(0)} /\left(4 \sqrt{17^{3}} \varsigma \alpha \Delta^{2}\right)$ at the threshold to a quenched turbulence regime, and thus the threshold condition can be written as

$\frac{\gamma}{v_{\mathbf{E} \times \mathbf{B}}^{\prime}} \simeq \frac{2 \Delta^{3 / 2}}{\chi \Lambda \sqrt{R T_{e, \max }}\left[1+13 \sqrt{14} k_{\Delta}^{2} L_{c} \chi_{j} \nu n_{\max }(0) \sqrt{T_{e, \max }(0)} \exp (-5 \delta / 2 \Delta) /\left(4 \sqrt{17^{3}} \varsigma \alpha \chi\right)\right]} \simeq 0.5$

Using Eqs. (33) and (34), the threshold condition in Eq. (35) can be rewritten as

$$
\begin{aligned}
& \frac{2 \Delta^{11 / 6}}{\chi \Lambda \sqrt{R}}\left(\frac{4 / 9 \Delta+2 \xi x_{S} / 3}{2 \pi L_{v} \int S_{T} d x}\right)^{1 / 3} \times \\
& \frac{136 \varsigma \alpha \Delta^{4} \chi\left(\xi x_{S}+2 \Delta / 3\right)}{136 \varsigma \alpha \Delta^{4} \chi\left(\xi x_{S}+2 \Delta / 3\right)+13(2 \pi)^{4} L_{v}^{2} \chi_{j} \nu \exp (-5 \delta / 2 \Delta) \int S_{n} d x} \simeq 0.5
\end{aligned}
$$

In Fig. 8, we plot the value of $\nu$ above which turbulence is quenched, as a function of $S_{0}$ for $\sigma=0.05$ and $\sigma=0.08$. In the same plot, the turbulence regime of the simulations we have performed is displayed. The agreement between the analytical estimate and simulation results is remarkable. 

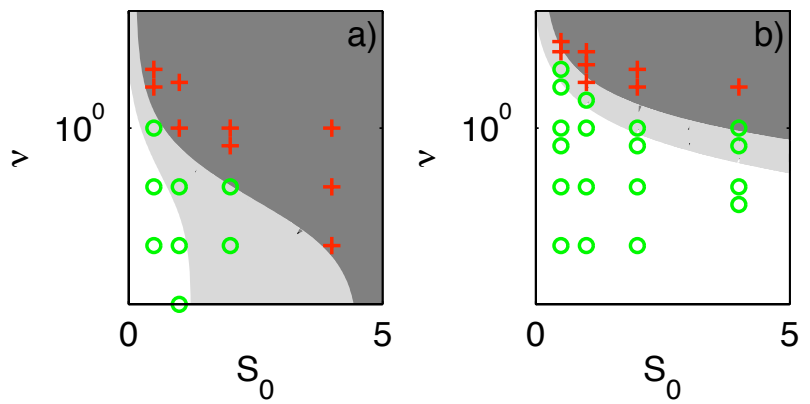

FIG. 8: (Color online) Threshold conditions for $\sigma=0.05$ (left, a) and $\sigma=0.08$ (right, b) as predicted from Eq. (36), dark gray corresponds to $\gamma / v_{\mathbf{E} \times \mathbf{B}}^{\prime}<0.4$ and light gray to $0.4<\gamma / v_{\mathbf{E} \times \mathbf{B}}^{\prime}<$ 0.6. Red ' + ' marks denote quenched turbulence simulations, green circles denote simulations in the $\mathrm{L}$ mode.

\section{DISCUSSION}

Within the flux tube model used here, the simulations we have explored display no $k_{\|} \neq 0$ regime; in particular, as shown by Eq. (36), the simulations are characterized by the onset of strong shear flow at either large plasma source $S_{T}$, or small value of the return distance of the magnetic field on the poloidal plane $\Delta$, or large parallel resistivity $\nu$. Shear flow quenches the turbulence for $\gamma / v_{\mathbf{E} \times \mathbf{B}}^{\prime}<0.5$. One can argue that the absence of drift-wave turbulence is a rather general result in the configuration adopted for the present simulations. Using the L-estimate for $L_{n}$, one can show that the growth rate of the drift wave over the interchange scales as $\left(R / L_{v}\right)^{1 / 3}$. However, according to Eq. (36), the decrease of $L_{v}$ and the increase of $R$ lead to a more rapid shear flow increase. Moreover, numerical tests show that shear flow is observed to steepen $L_{n}$ and $L_{T}$ to the source scale lengths. By reducing the plasma source scale length, it is thus possible to steepen the plasma profile, increasing the drift-wave linear growth rate. However, since the drift-wave linear growth rate scales with the inverse of $L_{n}$, while shear flow scales with the inverse of $L_{\phi}^{2}$, drift-wave turbulence remains shear-flow suppressed.

Experimental observations in TORPEX at small $\sigma$ display behavior that seems at first sight to be different from that of the flux tube simulations; at small $\sigma$ the experiments appear to display global convective cells instead of a strong shear flow, and instabilities with $k_{\|} \neq 0$ contribute to the plasma dynamics [5]. Future investigations are necessary in order 
to explain this apparent discrepancy, in particular we believe global simulations that take into account the impact of the vessel walls on the perpendicular dynamics are needed.

Here, we estimate the assumptions under which the $y$ periodic boundary conditions can be used: (i) $\tau / \tau_{f l}<1$, where $\tau$ is the time scale of the plasma dynamics and $\tau_{f l}$ is the flight time of the plasma, with $\tau_{f l}=L_{v} / v_{\mathbf{E} \times \mathbf{B}, y}$; (ii) $v_{\mathbf{E} \times \mathbf{B}, y} R /\left(c_{s} \sigma L_{v}\right)<1$, i.e., no global vortex formation, which is given by the fact that the plasma flowing along the vertical direction, $n v_{\mathbf{E} \times \mathbf{B}, z}$, is dissipated through parallel losses, $n c_{s} \sigma L_{v} / R$; and (iii) $L_{v} \int_{0}^{x_{S}} S_{n} d x / \int_{0}^{x_{S}} n v_{\mathbf{E} \times \mathbf{B}, y} d x>1$ and $L_{v} \int_{x_{S}}^{L_{x}} S_{n} d x / \iint_{x_{S}}^{L_{x}} n v_{\mathbf{E} \times \mathbf{B}, y} d x>1$, i.e. that the amount of plasma carried along the vertical direction at the left or right side of $x_{S}$, given by $\int_{0}^{x_{S}} n v_{\mathbf{E} \times \mathbf{B}, y} d x$ and $\int_{x_{S}}^{L_{x}} n v_{\mathbf{E} \times \mathbf{B}, y} d x$, can be provided by the plasma source, in the same domain, $L_{v} \int_{0}^{x_{S}} S_{n} d x$ and $L_{v} \int_{x_{S}}^{L_{x}} S_{n} d x$.

\section{Acknowledgments}

We gratefully acknowledge many useful discussions with A. Fasoli, I. Furno, B. Labit, and C. Theiler, as well as S. Brunner and T.M. Tran for their help in the parallelization of the code used here, and also M. Podestà for the careful reading of the manuscript. Computations were performed on the Pleiades clusters of the École Polytechnique Fédérale de Lausanne. P.R. was supported by a EURATOM fusion fellowship. B.R. acknowledges support by the USDOE.

\section{Appendix A: Brief summary of the main findings in the interchange dominated} regime

We briefly summarize the findings of Ref. [6] that examines configurations where the interchange mode is assumed to dominate over the drift-wave dynamics. Since $k_{\|}=0$, Eqs. (1-5) can be integrated along $z$, with the assumption that $\left.n V_{\| i}\right|_{z= \pm L_{c} / 2} \simeq \pm n \sqrt{T_{e}} /\left.2\right|_{z=0}$, and $\left.n V_{|| e}\right|_{z= \pm L_{c} / 2} \simeq \pm n \sqrt{T_{e}} \exp \left(\Lambda-\phi / T_{e}\right) /\left.2\right|_{z=0}$. One obtains

$$
\begin{aligned}
\frac{\partial n}{\partial t} & =R[\phi, n]+2\left(n \frac{\partial T_{e}}{\partial y}+T_{e} \frac{\partial n}{\partial y}-n \frac{\partial \phi}{\partial y}\right) \\
& +D \nabla^{2} n-\sigma n \sqrt{T_{e}} \exp \left(\Lambda-\phi / T_{e}\right)+S_{n}
\end{aligned}
$$




$$
\begin{gathered}
\frac{\partial \nabla^{2} \phi}{\partial t}=R\left[\phi, \nabla^{2} \phi\right]+2\left(\frac{T_{e}}{n} \frac{\partial n}{\partial y}+\frac{\partial T_{e}}{\partial y}\right) \\
+\nu \nabla^{4} \phi+\sigma \sqrt{T_{e}}\left[1-\exp \left(\Lambda-\phi / T_{e}\right)\right], \\
\frac{\partial T_{e}}{\partial t}=R\left[\phi, T_{e}\right]+\frac{4}{3}\left(\frac{7}{2} T_{e} \frac{\partial T_{e}}{\partial y}+\frac{T_{e}^{2}}{n} \frac{\partial n}{\partial y}-T_{e} \frac{\partial \phi}{\partial y}\right) \\
+k_{e} \nabla^{2} T_{e}-\frac{2}{3} \sigma \sqrt{T_{e}^{3}}\left[1.71 \exp \left(\Lambda-\phi / T_{e}\right)-0.71\right]+S_{T},
\end{gathered}
$$

We focus on the regime far from marginal stability and where the dominant instability has $k_{y}=k_{\Delta}$ [11]. In this regime, $L_{n} \ll R$ and the leading order terms in Eq. (A1) are $\partial_{t} n-R[\phi, n] \simeq 0$ from which, in the absence of a strong shear flow, one has $\partial_{y} \tilde{\phi} \sim$ $\gamma_{0} \tilde{n} /\left(R \partial_{x} \bar{n}\right)$ with $\gamma_{0}=\sqrt{2 T_{e} R / L_{p}}$. Turbulence saturation occurs for $\partial_{x} \bar{n}=\partial_{x} \tilde{n}$. The $\partial_{x} \tilde{n}$ term can be estimated by nonlocal linear theory [14], obtaining $\tilde{n} \sim \bar{n}\left(L_{p} / k_{\Delta}\right)^{1 / 2} / L_{n}$.

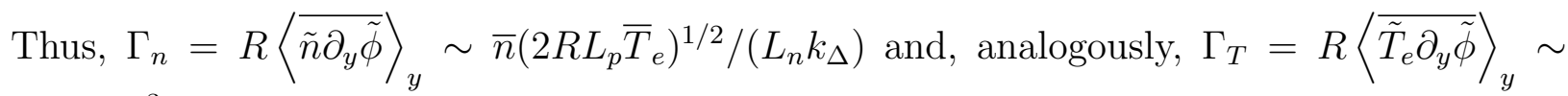
$\left(2 R L_{p} \bar{T}_{e}^{3}\right)^{1 / 2} /\left(L_{n} k_{\Delta}\right)$. The expression for $\Gamma_{n}$ and $\Gamma_{T}$ can be inserted into the $y$ - and timeaveraged Eq. (A1) and Eq. (A3)

$$
\frac{\partial \Gamma_{n}}{\partial x} \simeq S_{n}-\sigma \bar{n} \bar{T}_{e}^{1 / 2}, \frac{\partial \Gamma_{T}}{\partial x} \simeq S_{T}-\frac{2}{3} \sigma \bar{T}_{e}^{3 / 2},
$$

thus obtaining $L_{n}=0.58\left(L_{v} \sqrt{R}\right)^{2 / 3}$ and $L_{T}=0.74\left(L_{v} \sqrt{R}\right)^{2 / 3}$. In Ref. [6], the presence of a turbulence regime, where shear flow limits the perpendicular transport was observed, denoted as high confinement mode (H-mode) regime. This turbulence regime appears when $\gamma_{0} / v_{\mathbf{E} \times \mathbf{B}}^{\prime}<0.5$. Shear flow is imposed by the KH instability, $v_{\mathbf{E} \times \mathbf{B}}^{\prime} \simeq \chi \phi_{\max } / \Delta^{2}[\mathrm{Eq} .(22)]$. At the threshold for the H-mode, $L_{T} \simeq L_{n} \simeq L_{\phi} \simeq \Delta$, and thus the L-H threshold occurs for $2 \Delta^{3 / 2} /\left(\chi \Lambda \sqrt{R T_{e, \max }}\right) \simeq 0.5$. The value of $T_{e, \max }$ can be estimated from a global balance of Eq. (A3), obtaining

$$
\frac{2 \Delta^{11 / 6}}{\chi \Lambda \sqrt{R}}\left(\frac{4 / 9 \Delta+2 \xi x_{S} / 3}{2 \pi L_{v} \int S_{T} d x}\right)^{1 / 3} \simeq 0.5
$$

[1] A. Fasoli, B. Labit, M. McGrath, S. H. Müller, G. Plyushchev, M. Podestà, and F. M. Poli, Phys. Plasmas 13, 055902 (2006).

[2] J.C. Perez, W. Horton, K. Gentle, W. L. Rowan, K. Lee, and R. B. Dahlburg, Phys. Plasmas 13, 032101 (2006); K.W. Gentle and H. Huang, Plasma Science and Technology 10, 284 
(2008); E.D. Zimmerman and S.C. Luckhardt, J. Fusion Energy 12, 289 (1993); K. Rypdal and S. Ratynskaia, Phys. Rev. Lett. 94, 225002 (2005); P.K. Sharma and D. Bora, Plasma Phys. Control. Fusion 37, 1003 (1995); C. Riccardi, M. Fontanesi, A. Galassi, and E. Sindoni, Plasma Phys. Control. Fusion, 36, 1791 (1994).

[3] P. Ricci, C. Theiler, A. Fasoli, I. Furno, B. Labit, S. H. Müller, M. Podestà, and F. M. Poli, Phys. Plasmas 16, 055703 (2009).

[4] B. Li, B.N. Rogers, P. Ricci, and K.W. Gentle, Plasma transport and turbulence in the Helimak: simulation and experiment, accepted for publications on Physics of Plasmas (2009).

[5] F.M. Poli, P. Ricci, A Fasoli, M. Podestà, Phys. Plasmas 15, 032104 (2008).

[6] P. Ricci, B.N Rogers, and S. Brunner, Phys. Rev. Lett. 100, 225002 (2008).

[7] A. Zeiler, J.F. Drake, and B. Rogers, Phys. Plasmas 4, 2134 (1997).

[8] C.Q. Yu, S. I. Krasheninnikov, and P. N. Guzdar, Phys. Plasmas 13, 042508 (2006).

[9] M. Podestà, A. Fasoli, B. Labit, M. McGrath, S.H. Müller, and F.M. Poli, Plasma Phys. Controlled Fusion 48, 1053 (2006).

[10] I. Furno, B. Labit, M. Podestà, A. Fasoli, S.H. Müller, F.M. Poli, P. Ricci, C. Theiler, S. Brunner, A. Diallo, J. Graves, Phys. Rev. Lett. 100, 055004 (2008).

[11] P. Ricci and B.N. Rogers, Phys. Plasmas 16, 062303 (2009).

[12] A. Arakawa, J. Comp. Physics 1, 119(1966).

[13] P. Ricci, B.N. Rogers, W. Dorland, and M.A. Barnes, Phys. Plasmas 13, 062102 (2006)

[14] B.N. Rogers and W. Dorland, Phys. Plasmas 12, 062511 (2005).

[15] D. D. Ryutov and R. H. Cohen, Contrib. Plasma Phys. 44, 168 (2004).

[16] N. Bisai, A. Das, S. Deshpande, R. Jha, P. Kaw, A. Sen, and R. Singh, Phys. Plasmas 11, 4018 (2004).

[17] D. Biskamp, Magnetic reconnection in Plasma (Cambridge University Press, Cambridge, England, 2000). 
Table I. Study of the dependence of $L_{n}$ and $L_{T}$ on $D=D_{n}=D_{T}=D_{\phi}=D_{V e}=D_{V i}$ and $\eta_{0}=\eta_{0 i}=\eta_{0 e}$ for the configuration with $S=1, \sigma=0.05$, and $\nu=0.1$.

\begin{tabular}{|c|c|c|c|c|c|}
\hline \multicolumn{6}{|c|}{$D=0.01 \eta_{0}=0.1 D=0.1 \eta_{0}=0.1 D=1 \eta_{0}=0.1 D=1 \eta_{0}=0.01 D=1 \eta_{0}=1$} \\
\hline$L_{n}$ & 37.7 & 36.4 & 34.7 & 38.2 & 33.8 \\
\hline$L_{T}$ & 47.2 & 44.2 & 43.4 & 46.5 & 42.2 \\
\hline
\end{tabular}

